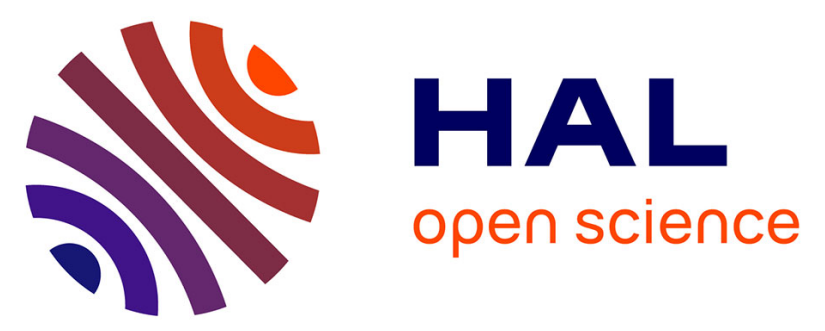

\title{
Grazing-angle reflectivity setup for the low-temperature infrared spectroscopy of two-dimensional systems
}

\author{
Marine Verseils, Alexandre Voute, Benjamin Langerome, Maxime Deutsch, \\ Jean-Blaise Brubach, Alexei Kalaboukhov, Alessandro Nucara, Paolo Calvani, \\ Pascale Roy
}

\section{To cite this version:}

Marine Verseils, Alexandre Voute, Benjamin Langerome, Maxime Deutsch, Jean-Blaise Brubach, et al.. Grazing-angle reflectivity setup for the low-temperature infrared spectroscopy of two-dimensional systems. Journal of Synchrotron Radiation, 2019, 26, pp.1945 - 1950. 10.1107/S1600577519010920 . hal-02884801

\section{HAL Id: hal-02884801 \\ https://hal.science/hal-02884801}

Submitted on 30 Jun 2020

HAL is a multi-disciplinary open access archive for the deposit and dissemination of scientific research documents, whether they are published or not. The documents may come from teaching and research institutions in France or abroad, or from public or private research centers.
L'archive ouverte pluridisciplinaire HAL, est destinée au dépôt et à la diffusion de documents scientifiques de niveau recherche, publiés ou non, émanant des établissements d'enseignement et de recherche français ou étrangers, des laboratoires publics ou privés. 
JOURNAL OF

SYNCHROTRON

RADIATION

ISSN 1600-5775

Received 28 May 2019

Accepted 4 August 2019

Edited by I. Lindau, SLAC/Stanford University, USA

\# Present address: Department of Chemistry, Technical University of Denmark, Kemitorvet Building 207, 2800 Kongens Lyngby, Denmark. $\S$ Present address: Université de Lorraine, CNRS, CRM2, F-54000 Nancy, France.

Keywords: infrared; grazing-angle reflectivity; low-temperature; two-dimensional systems.

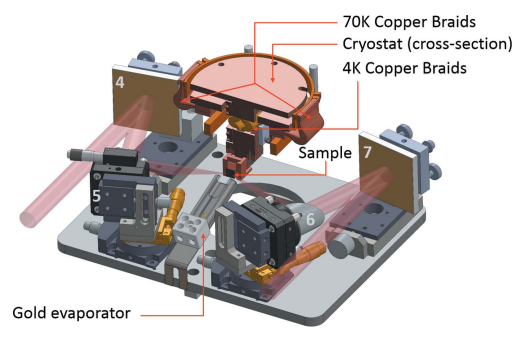

(C) 2019 International Union of Crystallography

\section{Grazing-angle reflectivity setup for the low-temperature infrared spectroscopy of two-dimensional systems}

\author{
Marine Verseils, ${ }^{\mathrm{a}}$ Alexandre Voute, ${ }^{\mathrm{a}} \neq$ Benjamin Langerome, ${ }^{\mathrm{a}}$ Maxime Deutsch, ${ }^{\mathrm{a}} \S$ \\ Jean-Blaise Brubach, ${ }^{\mathrm{a}}$ Alexei Kalaboukhov, ${ }^{\mathrm{b}}$ Alessandro Nucara, ${ }^{\mathrm{c}}$ Paolo Calvani ${ }^{\mathrm{c}}$ \\ and Pascale Roy ${ }^{\mathrm{a} *}$
}

\footnotetext{
${ }^{\mathbf{a}}$ Synchrotron SOLEIL, St Aubin, 91192 Gif-sur-Yvette, France, ${ }^{\mathbf{b}}$ Department of Microtechnology and Nanoscience, Chalmers University, S-41296 Gothenburg, Sweden, and ${ }^{\mathrm{c} C N R-S P I N}$ and Dipartimento di Fisica, Università di Roma La Sapienza, 00185 Roma, Italy. *Correspondence e-mail: pascale.roy@synchrotron-soleil.fr
}

A new optical setup is described that allows the reflectivity at grazing incidence to be measured, including ultrathin films and two-dimensional electron systems (2DES) down to liquid-helium temperatures, by exploiting the Berreman effect and the high brilliance of infrared synchrotron radiation. This apparatus is well adapted to detect the absorption of a 2DES of nanometric thickness, namely that which forms spontaneously at the interface between a thin film of $\mathrm{LaAlO}_{3}$ and its $\mathrm{SrTiO}_{3}$ substrate, and to determine its Drude parameters.

\section{Introduction}

Within the solid-state community, interest in the properties of ultrathin conducting films has rapidly grown in the last decades with the implementation of a variety of heterostructures, and nanoribbons of nanometric thickness, which have also shown interesting quantum effects related to electron confinement. An extreme case is represented by the twodimensional electron systems (2DES), where, in principle, the thickness $d$ of the conducting layer goes to zero. Well known examples are the topological surface states of topological insulators (Hasan \& Kane, 2010; Moore, 2010) and the 2DES at the interface between two insulators like $\mathrm{LaAlO}_{3}$ and $\mathrm{SrTiO}_{3}$ (LAO/STO) (Ohtomo et al., 2002; Ohtomo \& Hwang, 2004). Quasi-2DES are also present in the promising graphene ultrathin films shaped in the form of nanoribbons for future electronics (Barone et al., 2006).

Fourier-tranform infrared spectroscopy (FTIR) is traditionally a powerful tool for studying the low-energy electrodynamics of conducting materials, as it measures, directly and independently, the charge density (through the plasma frequency) and the scattering rate of the Fermi liquid (Dressel \& Grüner, 2002). However, its application to systems of nanometric thickness is generally prevented by their low specific absorbed intensity, which increases exponentially with $d$ and thus becomes vanishingly small in ultrathin films and in 2DES. The problem can be solved by replacing a conventional reflectivity setup for quasi-normal incidence measurements by an apparatus for grazing-angle spectroscopy with polarized radiation. In this way, one can exploit the Berreman effect (Berreman, 1963), a powerful spectroscopic tool that has been successfully used to study the 2DES (Dubroka et al., 2010; Yazdi-Rizi et al., 2017; Nucara et al., 2018) of LAO/STO and topological insulator systems like $\mathrm{Bi}_{2} \mathrm{Se}_{3}$ (Falsetti et al., 2018). 
In the Berreman effect, the $p$-component (in the plane of incidence) of the electric field $\mathbf{E}$ undergoes enhanced absorption through a film of thickness $d$ (if $d$ is much smaller than both the radiation wavelength $\lambda$ and the field penetration depth $d_{\mathrm{p}}$ ) at frequencies $\omega$ close to zero in the real part of the system dielectric function $\varepsilon_{1}(\omega)$. Therein, indeed, the normal component $E_{\mathrm{n}}^{a, f}(\omega)=D_{\mathrm{n}}^{f}(\omega) / \varepsilon_{1}(\omega)$, where $D_{\mathrm{n}}^{f}(\omega)$ is the normal component of the electric displacement field, becomes much larger than when in vacuum. Thus, in a film thinner than both $\lambda$ and $d_{\mathrm{p}}, E_{\mathrm{n}}(\omega)$ creates a dynamical dipole moment which resonates with the longitudinal excitations of the sample (Harbecke et al., 1985). By use of the Fresnel formulas for the optical response of a multilayer, one then obtains the parameters of the ultrathin film, namely, if it is metallic, the plasma frequency $\omega_{\mathrm{p}}$, the carrier relaxation rate $\Gamma$ and its thickness $d$.

In order to determine these parameters, it is necessary to implement an optical device suitable to perform reflectivity measurements at high angles of incidence, so that the electric field of the incident radiation has a strong $p$ component normal to the surface. As this increases the area of the illuminated sample, one needs to use a highly brilliant source, which moreover should be broad-band to allow for FTIR spectroscopy, properties being both characteristic of infrared synchrotron radiation (Roy et al., 2001). Finally, a compelling requirement of solid-state studies is that the sample can be cooled down to liquid-helium temperatures. Therefore, the grazing-angle optics must be adapted to a cryogenic apparatus, which can be placed in the sample compartment of the interferometer, or in an external vacuum chamber. These constraints do not allow a commercial optical device to be used. Because of its complexity, such a combination is scarce (see, for example, Stanislavchuk et al., 2013). In the present paper, we describe the apparatus built at one of the experimental stations of the infrared synchrotron beamline AILES at the facility SOLEIL. We shall also describe the measurements successfully performed on the 2DES at the LAO/STO interface. For the measurements of the Berreman effect, we used a single polarizer placed on the incident radiation path. However, this apparatus can also be easily adapted to ellipsometry measurements by the addition of a second polarizer - the analyzer - which intercepts the reflected radiation, and of two compensators on the beam path as considered by Yazdi-Rizi et al. (2017).

\section{Specifications and constraints}

As mentioned above, the purpose of the new apparatus is to perform reflectivity measurements at high incidence angles, with variable polarization, on samples mounted on the closedcycle cryogenerator whose cold finger is thermically connected to the sample holder. The cooled sample is placed at the center of an in-house-developed chamber external to the interferometer (Bruker IFS 125HR), referred to as the Cryostat Compartment (CC). The original Back Sample Compartment of the interferometer (BSC) is used here to host the optics which redirects the radiation to the $\mathrm{CC}$. The operating temperature range is $7-300 \mathrm{~K}$, and the position of the sample holder can be finely adjusted to compensate for any contraction at low temperature. The chamber, of dimensions $262 \mathrm{~mm}$ $\times 184 \mathrm{~mm} \times 162 \mathrm{~mm}$, can be kept at the same high vacuum $\left(P<10^{-6} \mathrm{mbar}\right)$ as the spectroscopic system or, alternatively, isolated by a set of windows adapted for each spectral range.

Requirements for the setup are (i) to focus the radiation on the sample at an angle of incidence of $72^{\circ} \pm 2^{\circ}$, (ii) to include a polarizer, and (iii) to take into account the dimensional constraints due to the chamber size and the cryostat position. It is designed to operate in the frequency range $10-3000 \mathrm{~cm}^{-1}$, using either the synchrotron radiation or the internal sources of the interferometer. In order to focus the entire synchrotron beam in the far-infrared domain, the sample has to be $5 \mathrm{~mm} \times$ $3 \mathrm{~mm}$ or larger. Furthermore, one can place in the sample compartment a mobile gold evaporator. This option allows a reliable reflectivity reference to be obtained when the sample is a single crystal, whose surface may still be rough after polishing, on the wavelength scale. Finally, the setup has to be user-friendly, given that the beamline AILES of SOLEIL is a facility open to researchers having limited beam time.

\section{Design and implementation of the optical setup}

Unlike other grazing-incidence setups where the cryostat vacuum chamber only contains the sample holder, our selected optical solution was based on inserting both the focusing optics and the sample holder in the same vacuum chamber. In addition to the $\mathrm{CC}$, a second assembly of optics is inserted in the BSC. A general schematic aerial view is shown in Fig. 1. Therein, for the sake of clarity, each mirror holds a number that will be used below to separately describe the optics in the two compartments.

\subsection{Optical assembly of the BSC}

A schematic of the BSC optical setup, before its insertion into the BSC, is shown in Fig. 2. In the description of the optical elements we shall refer to the numbers shown in Fig. 1.

The BSC optical assembly is mounted on an aluminium plate. It includes four flat mirrors $(1,3,8$ and 10) with $20 \mathrm{~mm}$ $\times 20 \mathrm{~mm}$ surface and a pair of paraboloid mirrors (2 and 9) with effective focal length $f=38.1 \mathrm{~mm}$. All mirrors are gold plated and have two screws on the back for the rotation around the vertical and horizontal axis allowing alignment. As shown in Fig. 1, a first focus point allowing insertion of optical elements such as polarizers, filters or iris is located between mirrors 1 and 2. The polarizer selects the electric field of the incident radiation either in the (horizontal) plane of incidence ( $p$ polarization) or orthogonally to it ( $s$ polarization). Mirror 2 is a paraboloid placed at the distance $f$ from the focus, to act as a collimator. The parallel beam is then sent to the flat mirror 3 , which deviates the beam toward the CC. The beam exits from the cryostat chamber and travels toward the detector through the other side of the BSC along a symmetric path through the flat (8 and 10) and the paraboloid (9) mirrors, which have the same $f$ as $2(38.1 \mathrm{~mm})$. In order to simplify the alignment, mirrors 1 and 10 are placed on the same rail which is parallel 


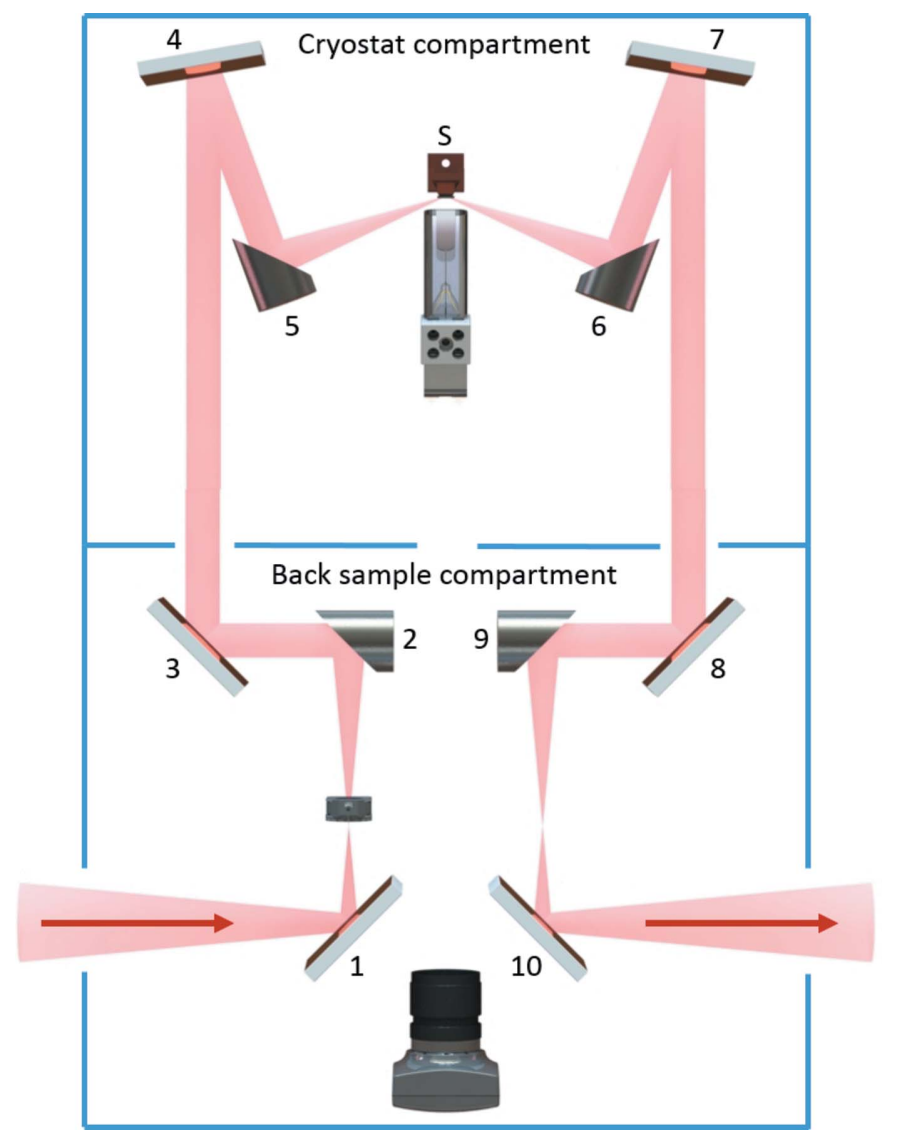

Figure 1

Schematic of the grazing-angle optical setup viewed from above. The modulated beam from the interferometer is focused and collimated by parabolic and flat mirrors numbered in the order of the optical path as described in the text. The focus point between mirrors 1 and 2 allows optical elements such as polarizers, filters or iris to be inserted. The position of the sample holder is indicated by the letter S. The camera at the bottom allows for checking the visible-light spot on the sample. The gold evaporator is below the camera axis.

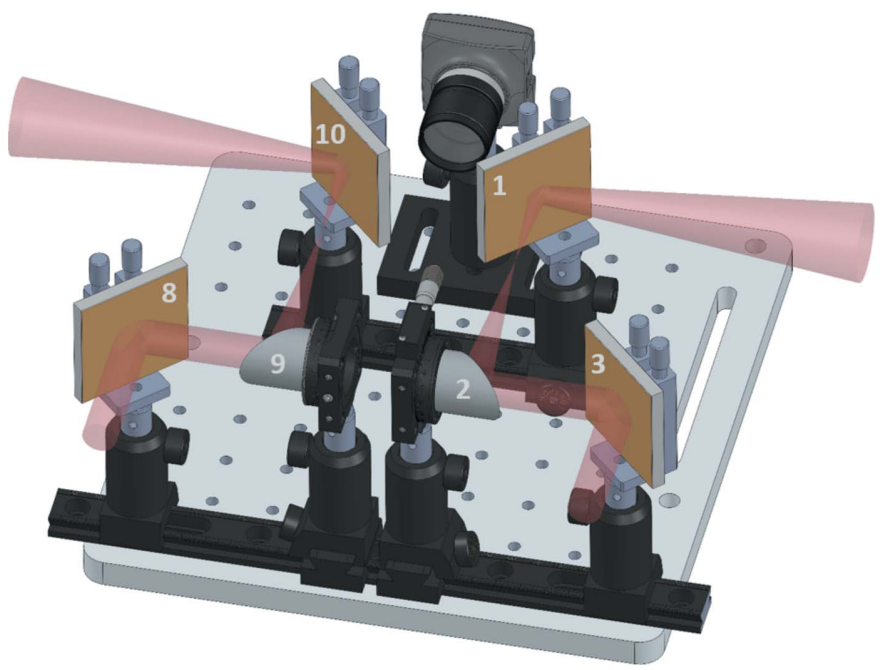

Figure 2

Schematic of the optical setup to be inserted in the back sample compartment. Mirrors 1, 3, 8 and 10 are flat. Mirrors 2 and 9 are paraboloids with focal length $f=38.1 \mathrm{~mm}$.

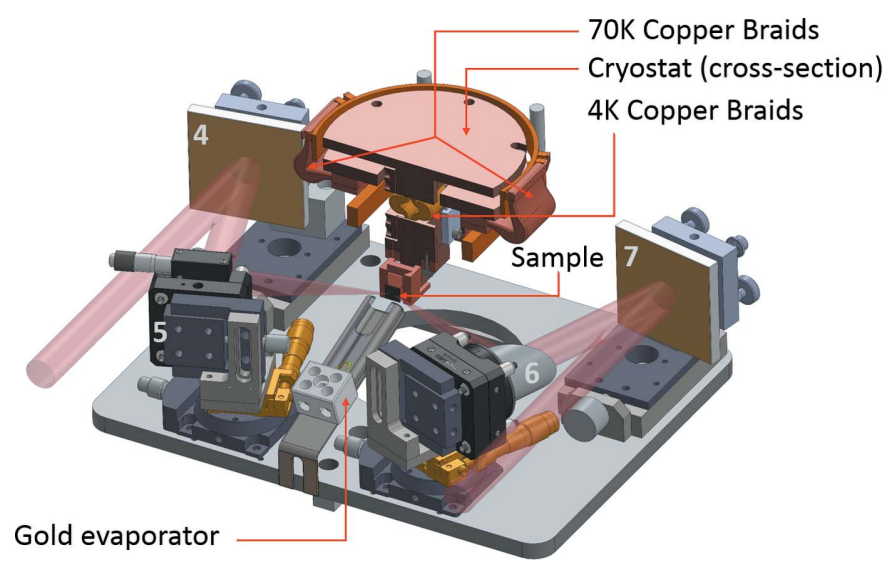

Figure 3

Schematic of the optical setup inserted in the cryostat compartment. Mirrors 4 and 7 are flat. Mirrors 5 and 6 are paraboloids mounted on a set of stages. The modulated beam is focused on the sample holder which is in thermal contact with the cryostat through copper braids. Note that the cold finger of the cryostat is not rigidly fixed to the sample holder in order to avoid large thermal contractions.

to the incident beam. Mirrors 3, 2, 9 and 8 are placed on a second rail, also parallel to the incident beam. Two screws are used for rotations around the vertical and horizontal axes. Both their height and the position along the rail are adjustable.

\subsection{Optical assembly of the CC}

A schematic of the second optical setup, taken before its insertion in the CC, is shown Fig. 3. Here also, the optical elements are identified by the numbers reported in Fig. 1. They are mounted on an aluminium board which holds a large aperture in order to allow for turbo pumping of the chamber. Flat mirror 4 deviates the parallel beam toward the paraboloid 5 , that focuses the beam onto the sample under an angle of $72^{\circ}$. The reflected radiation is collected and collimated again by paraboloid 6 , while flat mirror 7 reflects the parallel beam back to the BSC toward the detector. Both 5 and 6 have $f=$ $38.1 \mathrm{~mm}$ and the same adjustment screws mentioned above for mirrors 2 and 9. Details of the mechanical mounting are given in the next section for both the flat and the parabolic mirrors.

\subsection{Details of the translation and rotation stages}

The parabolic mirrors are mounted onto a stacking of small translation and rotation stages shown in Fig. 4, in order to fulfil the operational constraints of the two chambers. In order to define the angle of incidence $i$, we adjust movement (1) (see Fig. 4), which rotates the mirror around the $z$-axis. The linear translation along $y$ (2) allows for centering the grazing beam onto the sample for a given $i$. A groove allows adjustment along $z$ (3), and a linear translation stage along $x$ (4) permits adjustment of the focusing of the beam onto the sample. Finally, a rotating stage is mounted on the $x$ translation stage in order to permit rotation around the $y$ axis (5). 


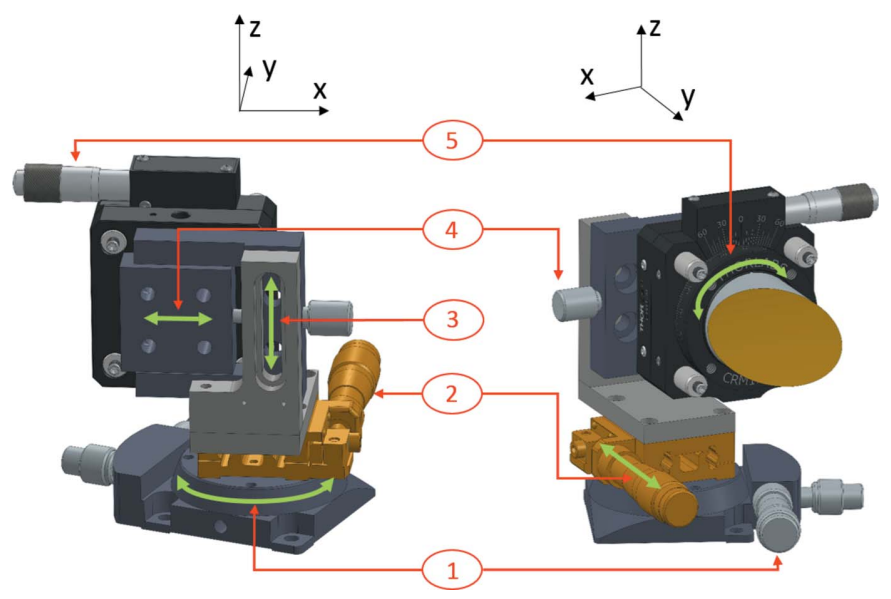

Figure 4

Schematic of the stages of rotation and translation used to hold and adjust the paraboloids 5 and 6 . Numbers correspond to the alignment controls as described in the text.

\subsection{Sample holder}

The surface of the sample is oriented toward the spectrometer. The sample holder, made of two oxygen-free, highconductivity (OFHC) copper pieces, as shown in Fig. 5, ensures a good thermal contact with the cold finger of the cryogenerator. The sample (2) is fixed on a truncated pyramid (2) with a cryogenic varnish in order to reject the 'excess' of radiation out of the optical path. Piece (1) is an adapter with a trapezoidal groove where the pyramid is placed. It has a square base, so that it can be inserted in two orthogonal directions. This can be useful for rotating the polarization on anisotropic samples by exploiting the full intensity of the highly linearly polarized radiation coming from the synchrotron port. The position into the groove is fixed by a lateral screw which also allows for a good thermal contact. In order to make the position of the sample reproducible, the back of the adapter is inserted into a tight slit providing an accurate positioning onto the cryostat and a good thermal contact.

\section{An application to the two-dimensional electron system at the LAO/STO interface}

As already reported in previous work (Roy et al., 2001, 2006; Voute et al., 2016), infrared spectroscopy takes much advan-

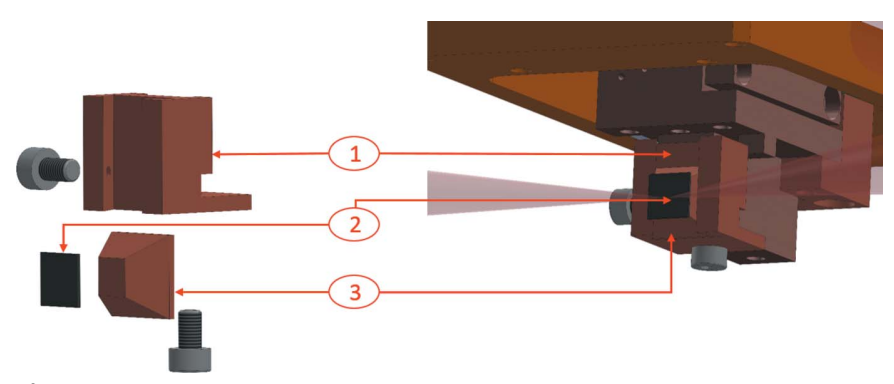

Figure 5

The sample holder: exploded view (left) and assembled (right): adaptor (1); sample (2); pyramid (3). tage from the high brilliance of synchrotron radiation with respect to conventional (blackbody) wide-band sources, especially in the far-infrared and the terahertz range. The gain in terms of signal-to-noise ratio, which is already remarkable in reflectivity measurements at normal incidence, increases even further with the angle of incidence. At grazing incidence, the advantage of using synchrotron radiation is important also in the mid-infrared up to $3000 \mathrm{~cm}^{-1}$ as shown in Fig 6 .

Such an advantage can be exploited both in ellipsometry and in the measurements based on the Berreman effect as mentioned in the Introduction. As an example of the latter application, measurements on $\mathrm{SrTiO}_{3} / \mathrm{LaAlO}_{3}$ thin films were performed at beamline AILES of Synchrotron SOLEIL with the aforementioned apparatus and are presented in the following.

At the interface between a $\mathrm{SrTiO}_{3}$ (STO) substrate and a $\mathrm{LaAlO}_{3}$ (LAO) film thicker than four unit cells, a thin layer of free charges was found to appear spontaneously (Ohtomo et al., 2002; Mannhart \& Schlom, 2010). Having a thickness certainly smaller than $10 \mathrm{~nm}$ (Basletic et al., 2008; Dubroka et al., 2010; Yazdi-Rizi et al., 2017; Nucara et al., 2018), it is considered as a 2DES. According to several authors (Ohtomo et al., 2002; Ohtomo \& Hwang, 2004; Caviglia et al., 2010), the 2DES forms within the upper layer of STO, through a topdown charge transfer triggered by the need to avoid a 'dipolar catastrophe' in LAO. According to others (Willmott et al., 2007; Kalabukhov et al., 2007), self-doping by oxygen nonstoichiometry at or around the interface can play a major role. This layer has been investigated by infrared spectroscopy combined with a full ellipsometric apparatus (Dubroka et al., 2010). It is therefore an ideal system to test our new setup using a single polarizer at SOLEIL. To this purpose, two samples of crystalline LAO films deposited on $\mathrm{TiO}_{2}$-terminated $\mathrm{SrTiO}_{3}$ substrates were prepared by pulsed laser deposition (Aurino et al., 2013). In sample $A$ the 2DES was present, as demonstrated by transport measurements (Nucara et al., 2018), while in sample $B$ - to be used as reference - the 2DES had been erased by ion etching. The reflectivity $R(\omega)$ of

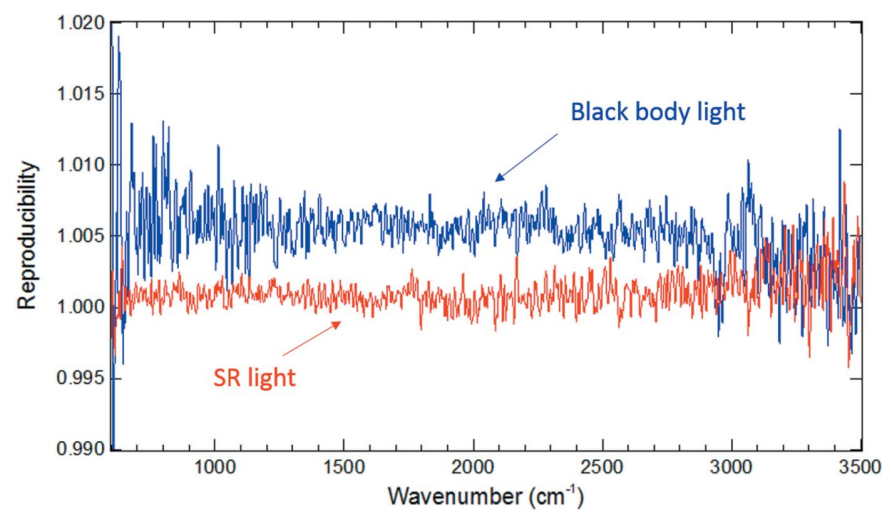

Figure 6

Mid-infrared reproducibility for synchrotron (SR) light compared with a blackbody (BB) source for the grazing-angle reflectivity setup available on the AILES beamline of Synchrotron SOLEIL. SR: red curve; BB: blue curve. Both curves were obtained under the same conditions of measurement: MCT detector, $1.5 \mathrm{~mm}$ iris opening, $\mathrm{KBr}$ beamsplitter and 400 scans. 


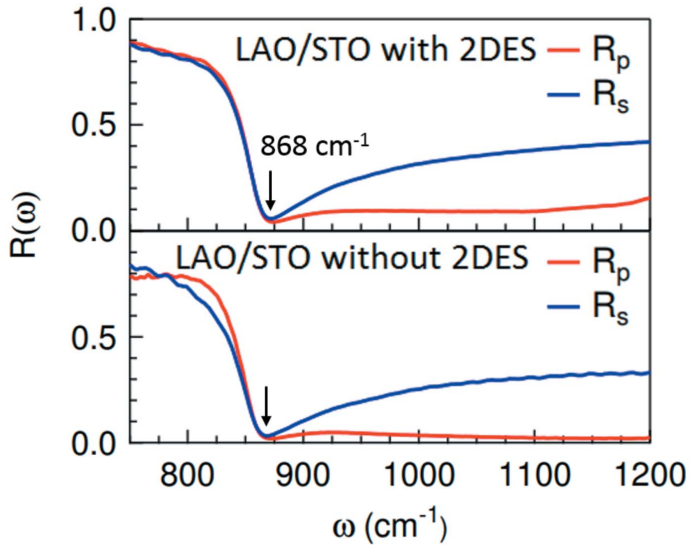

Figure 7

Reflectivity of LAO/STO with 2DES (top) (sample $A$ ) and without 2DES (bottom) (sample $B$ ), measured at $T=10 \mathrm{~K}$ with the radiation parallel $\left(R_{p}\right)$ and orthogonal $\left(R_{s}\right)$ to the plane of incidence of the infrared radiation. The resonance is observed on the dip of the reflectivity edge of the longitudinal phonon as indicated by the arrows. For both samples the reference was a gold mirror.

$A$ and that of $B$ were measured independently, firstly with the electric field parallel to the plane of incidence $\left[R_{p}(\omega)\right]$ and then orthogonally to it $\left[R_{s}(\omega)\right]$, see Fig. 7 . We thus extracted for both samples the ellipsometric angle

$$
\Psi(\omega)=\arctan \left(R_{p} / R_{s}\right)^{1 / 2},
$$

and finally the difference $\Delta \Psi=\Psi_{A}-\Psi_{B}$, at various temperatures, as plotted in Fig. 8. Therein, one observes a Berreman resonance at $868 \mathrm{~cm}^{-1}$, which is better defined at low temperature. This frequency corresponds to the reflectivity edge of a longitudinal phonon of STO, as predicted by theory (Berreman, 1963; Dubroka et al., 2010). Thanks to this resonance, we could isolate the spectral contribution of the 2DES at the interface. The refraction index for the no-2DES sample (sample $B$ ) is obtained with the Lyddane-Sachs-Teller (LST) equation which allows the dielectric function of the

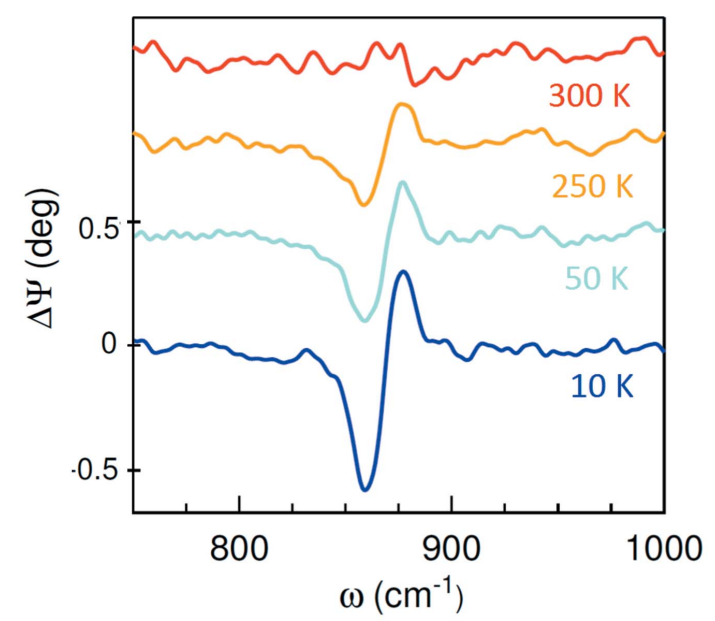

Figure 8

Contribution to the Berreman effect of the 2DES at the interface between crystalline LAO and STO, as obtained by subtracting from the ellipsometric angle $\Psi(\omega)$ of sample $A$ (with the 2DES) that of sample $B$ where the 2DES had been erased by ion etching.
Table 1

Fitting parameters for the 2DES at the interface between a crystalline LAO film and its STO substrate: 2DES thickness $(d)$, carrier plasma frequency $\omega_{\mathrm{p}}$ and relaxation rate $\Gamma_{D}$.

\begin{tabular}{cccc}
\hline$T(\mathrm{~K})$ & $d(\mathrm{~nm})$ & $\omega_{\mathrm{p}}\left(\mathrm{cm}^{-1}\right)$ & $\Gamma_{D}\left(\mathrm{~cm}^{-1}\right)$ \\
\hline 250 & $4 \pm 1$ & $980 \pm 80$ & $155 \pm 20$ \\
10 & $4 \pm 1$ & $950 \pm 80$ & $5 \pm 2$ \\
\hline
\end{tabular}

Table 2

Comparison of our values of sheet carrier density $N_{\mathrm{s}}$ and mobility $\mu$ with those reported by Dubroka et al. (2010) in a previous work.

\begin{tabular}{ll}
\hline Dubroka et al. & Our work \\
\hline$N_{\mathrm{s}}(300 \mathrm{~K})=6 \pm 1 \times 10^{13} \mathrm{~cm}^{-2}$ & $N_{\mathrm{s}}(250 \mathrm{~K})=1.4 \pm 0.3 \times 10^{13} \mathrm{~cm}^{-2}$ \\
$N_{\mathrm{s}}(\sim 10 \mathrm{~K})=9 \pm 1 \times 10^{13} \mathrm{~cm}^{-2}$ & $N_{\mathrm{s}}(10 \mathrm{~K})=1.3 \pm 0.3 \times 10^{13} \mathrm{~cm}^{-2}$ \\
$\mu(300 \mathrm{~K}) \simeq 3 \mathrm{~cm}^{2} \mathrm{~V}^{-1} \mathrm{~s}^{-1}$ & $\mu(250 \mathrm{~K})=20 \pm 2 \mathrm{~cm}^{2} \mathrm{~V}^{-1} \mathrm{~s}^{-1}$ \\
$\mu(\sim 10 \mathrm{~K})=600 \pm 300 \mathrm{~cm}^{2} \mathrm{~V}^{-1} \mathrm{~s}^{-1}$ & $\mu(10 \mathrm{~K})=580 \pm 40 \mathrm{~cm}^{2} \mathrm{~V}^{-1} \mathrm{~s}^{-1}$ \\
\hline
\end{tabular}

substrate to be expressed as a function of the ratio of the longitudinal and transverse phonon frequencies. In turn, the refractive index in the sample with $2 \mathrm{DES}$ (sample $A$ ) is obtained by adding to the LST expression, a free-electron Drude term. Then, the ellipsometric angle is expressed as a function of the reflection coefficient of the entire multilayer from the so-called Fresnel equations, taking into account that the two interfaces of the 2DES are both transmitting and reflecting, and is fitted with the experimental data. For more details and exact equations see Nucara et al. (2018). We obtained its physical and electrodynamic parameters, namely the thickness $d$ of the 2DES, the plasma frequency $\omega_{\mathrm{p}}$ and the electron relaxation rate $\Gamma_{D}$. The resulting Drude parameters are summarized in Table 1 . From these parameters we can deduce the sheet carrier density $N_{\mathrm{s}}$ and the mobility $\mu$ at 250 and $10 \mathrm{~K}$. In Table 2 we compare these values with those reported by Dubroka et al. (2010). Our samples were not obtained with the same annealing procedure and it is known that the charge density depends on the oxygen concentration at the interface which is sample dependent. In view of the above considerations, we can conclude that our values are consistent with the previous work showing that the apparatus here described, in combination with a synchrotron source, is fully adapted for this kind of measurement.

\section{Conclusion}

We have described an optical setup for reflectivity measurements at grazing incidence which can work both with the internal sources of the interferometer and, more efficiently, with infrared synchrotron radiation, down to the far-infrared range. Moreover, the sample can be temperature controlled down to $7 \mathrm{~K}$. It has been tested to investigate the electrodynamic properties of two-dimensional conducting systems by use of the Berreman effect, but it can also be applied to the spectroscopy of other ultrathin films, like for instance those formed by molecules adsorbed on surfaces. With a few changes, the apparatus can also be used for ellipsometry or 
photo-modulated infrared reflection absorption spectroscopy (Buffeteau et al., 1991). In a first test of the optical setup, we detected the Berreman resonance of a $2 \mathrm{DES}$ of nanometric thickness, formed spontaneously at an LAO/STO interface. We obtained results similar to those of a previous experiment performed with an ellipsometric apparatus (Dubroka et al., 2010). We could determine the Drude parameters of the 2DES which is only $4 \mathrm{~nm}$ thick. Such a setup is also well adapted for studying further 2DES like the topological surface states (Falsetti et al., 2018) or graphene-based systems.

\section{Funding information}

Funding for this research was provided by: Agence Nationale de la Recherche (grant No. ANR-10-LABX-0039); and Ministero dell'Istruzione, dell'Università e della Ricerca.

\section{References}

Barone, V., Hod, O. \& Scuseria, G. E. (2006). Nano Lett. 6, 2748-2754. Basletic, M., Maurice, J.-L., Carrétéro, C., Herranz, G., Copie, O., Bibes, M., Jacquet, E., Bouzehouane, K., Fusil, S. \& Barthélémy, A. (2008). Nat. Mater. 7, 621-625.

Berreman, D. (1963). Phys. Rev. 130, 2193-2198.

Buffeteau, T., Desbat, B. \& Turlet, J. M. (1991). Appl. Spectrosc. 45, 380-389.

Caviglia, A. D., Gariglio, S., Cancellieri, C., Sacépé, B., Fête, A., Reyren, N., Gabay, M., Morpurgo, A. F. \& Triscone, J. M. (2010). Phys. Rev. Lett. 105, 236802.

Dressel, M. \& Grüner, G. (2002). Electrodynamics of Solids. Cambridge University Press.

Dubroka, A., Rössle, M., Kim, K. W., Malik, V. K., Schultz, L., Thiel, S., Schneider, C. W., Mannhart, J., Herranz, G., Copie, O., Bibes, M., Barthélémy, A. \& Bernhard, C. (2010). Phys. Rev. Lett. 104, 156807.
Falsetti, E., Nucara, A., Shibayev, P., Salehi, M., Moon, J., Oh, S., Brubach, J.-B., Roy, P., Ortolani, M. \& Calvani, P. (2018). Phys. Rev. Lett. 121, 176803.

Harbecke, B., Heinz, B. \& Grosse, P. (1985). Appl. Phys. A, 38, $263-$ 267.

Hasan, M. Z. \& Kane, C. L. (2010). Rev. Mod. Phys. 82, 3045-3067.

Kalabukhov, A., Gunnarsson, R., Börjesson, J., Olsson, E., Claeson, T. \& Winkler, D. (2007). Phys. Rev. B, 75, 121404.

Mannhart, J. \& Schlom, D. (2010). Science, 327, 1607-1611.

Moore, J. E. (2010). Nature, 464, 194-198.

Nucara, A., Corasaniti, M., Kalaboukhov, A., Ortolani, M., Falsetti, E., Sambri, A., Miletto Granozio, F., Capitani, F., Brubach, J.-B., Roy, P., Schade, U. \& Calvani, P. (2018). Phys. Rev. B, 97, 155126.

Ohtomo, A. \& Hwang, H. (2004). Nature, 427, 423-426.

Ohtomo, A., Muller, D. A., Grazul, J. L. \& Hwang, H. Y. (2002). Nature, 419, 378-380.

Paolo Aurino, P., Kalabukhov, A., Tuzla, N., Olsson, E., Claeson, T. \& Winkler, D. (2013). Appl. Phys. Lett. 102, 201610.

Roy, P., Brubach, J.-B., Calvani, P., De Marzi, G., Filabozzi, A., Gerschel, A., Giura, P., Lupi, S., Marcouié, O., Mermet, A., Nucara, A., Paolone, A. \& Vervloet, M. (2001). Nucl. Instrum. Methods Phys. Res. A, 467-468, 426-436.

Roy, P., RouzièRes, M., Qi, Z. \& Chubar, O. (2006). Infrared Phys. Technol. 49, 139-146.

Stanislavchuk, T., Kang, T., Rogers, P., Standard, E., Basistyy, R., Kotelyanskii, A., Nita, G., Zhou, T., Carr, G., Kotelyanskii, M. \& Sirenko, A. A. (2013). Rev. Sci. Instrum. 84, 023901.

Voute, A., Deutsch, M., Kalinko, A., Alabarse, F., Brubach, J.-B., Capitani, F., Chapuis, M., Ta Phuoc, V., Sopracase, R. \& Roy, P. (2016). Vib. Spectrosc. 86, 17-23.

Willmott, P. R., Pauli, S. A., Herger, R., Schlepütz, C. M., Martoccia, D., Patterson, B. D., Delley, B., Clarke, R., Kumah, D., Cionca, C. \& Yacoby, Y. (2007). Phys. Rev. Lett. 99, 155502.

Yazdi-Rizi, M., Marsik, P., Mallett, B. P. P., Sen, K., Cerreta, A., Dubroka, A., Scigaj, M., Sánchez, F., Herranz, G. \& Bernhard, C. (2017). Phys. Rev. B, 95, 195107. 